\title{
Optimal Design of Experimental Epidemics
}

\author{
D.E. Pagendam*, P.K. Pollett** \\ ${ }^{a}$ CSIRO Mathematics, Informatics and Statistics, 120 Meiers Rd, Long Pocket QLD \\ 4068, Australia \\ ${ }^{b}$ Department of Mathematics, University of Queensland, St Lucia QLD 4072, Australia
}

\begin{abstract}
We consider the optimal design of experimental epidemics modelled as density dependent Markov processes. We focus on finding (i) the optimal times at which to collect data about the state of the system for a small number of discrete observations, (ii) the optimal numbers of susceptible and infective individuals to begin an experiment with, and (iii) the optimal number of replicate epidemics to use. We answer these questions by looking at three commonly used compartmental models in experimental epidemiology: the SI (Susceptible to Infected), SIS (Susceptible to Infected to Susceptible) and partially observed SIR (Susceptible to Infected to Recovered) epidemics. In particular, to demonstrate the wide range of density dependent models to which our methodology can be applied, we use a time-homogeneous SIS epidemic, a time-inhomogeneous SI epidemic with exponentially decreasing transmission rates and an partially observed SIR epidemic where the infectious period for an individual has a gamma distribution. Whilst compartmental models are
\end{abstract}

\footnotetext{
*Principal corresponding author

** Corresponding author

Email addresses: dan.pagendam@csiro.au (D.E. Pagendam), pkp@maths.uq.edu.au (P.K. Pollett)
} 
widely applied in natural systems, we focus on the use of the models in the context of controlled experimental epidemics or transmission experiments, whose purpose is to inform the practitioner about disease transmission and recovery rates. We adopt the popular $D$-optimality criterion as providing an appropriate objective function for designing our experiments, since this leads to point estimates with maximum precision. As $D$-optimal designs for non-linear models are only locally optimal, we also consider the robustness of the designs relative to the misspecification of the model parameters.

Keywords: Markov chain; optimal design; Fisher information; sampling; SIS; SIR; SI; SI(k)R; epidemic.

\section{Introduction}

Transmission experiments are controlled experimental epidemics involving populations of diseased and healthy organisms, often contained in a microcosm or conducted in a laboratory. The objective is to observe, over time, the spread of disease amongst the organisms and to use this information to obtain point estimates of per capita transmission and recovery rates. In recent years, such experiments have been used to study the dynamics of footand-mouth disease (Streftaris and Gibson, 2004), avian influenza (van der Goot et al., 2005), damping off (Kleczkowski et al., 1997; Cook et al., 2008; Otten et al., 2003) and Belgian classical swine fever (Höhle et al., 2005). Some of the common epidemiological models used for studying the spread of disease among interacting organisms include the SI (susceptible to infected), SIS (susceptible to infected to susceptible) and SIR (susceptible to infected to recovered) epidemics, and variants thereof (see for example Stone et al. 
(2008), McCormack and Allen (2007) and Kim and Radhakrishnan (2006)).

They are examples of the compartmental epidemic models introduced initially by Kermack and McKendrick (1927) as deterministic models and later popularized as stochastic processes by Bartlett (1949, 1956), Bailey (1950, 1957) and Kendall (1956). Many belong to a broader class of birth-death processes or Markov population processes (Kingman, 1969) known as density dependent Markov processes (DDMPs), as identified and studied by Kurtz (1970), and to which the methodology, herein, applies.

Early work on inference for birth-death processes either considered scenarios where a process was observed continuously in time (Keiding, 1974, 1975) or observed at equally spaced times (Keiding, 1975; Kendall, 1949). These simplifications were necessary to obtain analytical expressions for maximum likelihood estimators. When dealing with discretely observed processes, there is often the complication that the transition probabilities are difficult to evaluate analytically and the likelihood cannot be written down in simple form. Such is the case when designing experiments for compartmental models such as the SI, SIS and SIR epidemics. Consequently, it becomes necessary to either evaluate probabilities numerically (for example using EXPOKIT (Sidje, 1998)), to rely on Monte Carlo methods (O'Neill and Roberts, 1999; Cauchemez and Ferguson, 2008) or to employ distributional approximations. Given the widespread use of compartmental models, and the degree of attention given to parameter estimation, it is surprising that little attention has been directed towards the design of efficient experiments. There is only a handful of contributions: Becker and Kersting (1983) examined the simple birth process, Cook et al. (2008) examined the simple death process 
and a time-inhomogeneous SI epidemic, and Pagendam and Pollett (2009, 2010b,a) examined the simple birth-death, the stochastic logistic model, and the simple death processes, respectively. In the models examined by Becker and Kersting (1983) and Pagendam and Pollett (2010a) there was a single parameter, and the model was sufficiently simple that optimal experimental designs could be obtained analytically. For multi-parameter models and some single parameter models (the SI epidemic for example), likelihoods are rarely in a form that allows calculation of the expected Fisher information matrix, which lies at the heart of most classical optimality criteria in experimental design.

The recent work of Cook et al. (2008) identified optimal observation times for an SI epidemic in a Bayesian framework. They relied on a method known as moment closure to approximate the likelihood function of their model. In essence, the transition kernel of their SI epidemic was approximated using a beta-binomial distribution as described by Krishnarajah et al. (2005), but choice of this particular distributional approximation was specific to their model. Many other distributions have been employed for moment closure: Whittle (1957) introduced the technique using the normal distribution; Keeling (2000) used the log-normal distribution; Nåsell (2003) used the Poisson, binomial and log-normal. (These methods have been compared recently by Clancy and Mendy (2011).) Here, we develop alternative methodology for optimal experimental design of DDMPs, as used in experimental epidemiology, which relies on Gaussian diffusion approximations. These offer a unified approach for rapidly obtaining designs for a wide range of single and multidimensional epidemiological models, provided that the system size (usually 
the total population size) is reasonably large. We pose the design problem in the classical frequentist framework and illustrate some important findings using time-inhomogeneous SI, time-homogeneous SIS and partially observed $\mathrm{SI}(\mathrm{k}) \mathrm{R}$ (having a gamma distributed infectious period) epidemics as examples.

In estimating the parameters of epidemic models, our aims are threefold: (i) to find the optimal times to observe the epidemic, (ii) to find the optimal number of infected organisms to introduce to a susceptible population, and (iii) to determine whether there is any difference between running one large experiment or several smaller ones. This is in contrast to previous work that has focussed on optimal observation times alone. Our problem can be framed in the context of optimal experimental design and we employ the popular D-optimality criterion (see Atkinson et al. (2007)). The optimal designs obtained are locally optimal in the sense of Chernoff (1953), since the choice of observation times and initial conditions of the process will vary depending on the parameter vector $\theta$. For the SI(k)R epidemic we may only use the $D$-optimal design for estimation of $\beta$ and $\gamma$ (the transmission and recovery rates) under the assumption that $k$ (the number of infectious classes) is known. This is because $k$ is discrete; the Fisher information is defined for continuous parameters. In the context of experimental epidemics, the choice of $k$ is an issue of model selection, rather than a parameter we wish to estimate. Whilst model selection is beyond the scope of this paper, we note that $T$-optimal designs and their variants can be used for this purpose, and that the methods and theory presented here is also applicable in that context. 


\section{The SI and SIS epidemic}

The SI and SIS epidemics are two closely related birth-death processes used for modelling epidemics where individuals make transitions from susceptible (disease free) to infected. In the case of the SIS epidemic, individuals can recover from the disease and transition back to the susceptible state so that reinfection may occur; for the SI epidemic recovery is impossible. Both models have state space $\mathcal{S}=\{0, \ldots, N\}$, representing the number of infected individuals. We consider SI and SIS epidemics with the following general form for the transition rates:

$$
q(i, i+l ; t)= \begin{cases}\frac{\beta e^{-\lambda t}}{N} i(N-i) & (l=1) \\ \mu i & (l=-1)\end{cases}
$$

where $N$ is the total population size, $i$ is the number of infected individuals, $\beta(>0)$ is the disease transmission rate, $\mu(\geq 0)$ is the individual recovery rate and $\lambda(>0)$ is a parameter governing an exponential decline in the transmission rate with time. The latter parameter can be used, for example, to account for the decrease in infection risk as an organism ages, sometimes referred to as quenching (see Bailey et al. (2000) for example). The transition rates of the SI epidemic we will consider are a special case of those above with $\mu=0$, so that the number of diseased organisms increases monotonically over time (a pure birth process). For both models, 0 is an absorbing state corresponding to the eradication of disease. Note, however, that the SI epidemic is absorbed only in the trivial situation where the epidemic begins with no infected organisms. The SI epidemic has a second absorbing state $N$ corresponding to the infection of every individual in the population. Clearly, 
once an experimental epidemic has reached an absorbing state, it provides no more information regarding the disease dynamics.

For the SIS epidemic, we shall restrict attention to the time-homogeneous case, where $\lambda=0$. A useful reparameterization of this model is $\alpha=\beta-\mu$ and $\rho=\mu / \beta$. As noted in previous work (Ross et al., 2006; Pagendam and Pollett, 2009), one advantage of using $(\alpha, \rho)$ is that there is much weaker dependence between the elements of the maximum likelihood estimate, $\hat{\boldsymbol{\theta}}$, than for $(\beta, \mu)$, greatly aiding visualization of the maximum likelihood estimator. A further rationale for using these parameters stems from their relationship with the phases of the SIS epidemic, as we now explain. Roughly speaking, the SIS epidemic has two phases (see Figure 1(b)): (i) an initial period of drift towards a stable equilibrium, and (ii) a period of fluctuation about this equilibrium. The rate at which the epidemic is attracted towards equilibrium is governed by $\alpha$ whilst the position of the equilibrium is determined by $\rho$. As we shall see, observing the process close to equilibrium provides more information about $\rho$ than $\alpha$, whilst the opposite is true in the drift phase. We emphasize, however, that these two phases do not exclusively provide information about each of the parameters independently, since, for example, $\alpha$ also governs the decay rate of the correlation between successive observations in both phases.

\section{The partially observed SI(k)R epidemic}

The general SI(k)R epidemic (see Ross et al. (2009)) is a generalization of the standard SIR model, but where we suppose that each individual passes through $k$ infectious stages (potentially with different parameters), where a 
susceptible individual may contract a disease through interaction with an infected individual, but can subsequently recover, acquiring immunity from further infection. Clearly, the standard SIR model presented in Andersson and Britton (2000) is equivalent to the SI(1)R model. We shall limit our focus to a slightly simpler version of the more general SI(k)R model, where the parameters in each of the infectious stages are identical and therefore the total time an individual is infectious for has a $\operatorname{Gamma}(k, \beta)$ distribution. We make special note however, that this model is flexible enough to allow the infectious period to have any phase-type distribution (Neuts, 1975) and that the diffusion approximation methodology outlined here will still be applicable. Let $S_{t}$ denote the number of susceptible individuals, $I_{1, t}, \ldots, I_{k, t}$ the numbers of infected individuals in $k$ stages of infection and $R_{t}$ the number of recovered (and immune) individuals in a closed population of size $N$. We impose the realistic constraint that we cannot distinguish between which of the $k$ (somewhat artificial) stages of infection an individual is in; only the total number of infected individuals, $I_{\text {Total }, t}=\sum_{j=1}^{k} I_{j, t}$ (the sum of individuals in each of the $k$ classes), can be observed at any given time. In addition, infected individuals are more easily identified since they are symptomatic. However, it can be more difficult to discern between susceptible and recovered individuals and measure their numbers. We therefore assume that the initial numbers in $S_{0}, I_{1,0}, \ldots, I_{k, 0}$ and $R_{0}$ are known, but that for all subsequent observations only $I_{\text {Total, } t}$ can be measured. We refer to this scenario as the partially observed $\mathrm{SI}(\mathrm{k}) \mathrm{R}$ (henceforth poSI $(\mathrm{k}) \mathrm{R}$ ) epidemic.

The state space $\mathcal{S}$ of the $\mathrm{SI}(\mathrm{k}) \mathrm{R}$ epidemic $\mathbf{Y}_{t}=\left(S_{t}, I_{1, t}, \ldots, I_{k, t}, R_{t}\right)$ is $(k+2)$-dimensional. However, in a closed population we need only account 
for the number susceptible and the numbers in the infected classes since $R_{t}=N-S_{t}-\sum_{i=1}^{k} I_{i, t}$. For an $\mathrm{SI}(\mathrm{k}) \mathrm{R}$ epidemic the state space can be expressed as

$$
\mathcal{S}=\left\{\left(s, i_{1}, \ldots, i_{k}, r\right) \in Z_{+}^{k+2}: s+i_{1}+\cdots+i_{k+r}=N\right\},
$$

having absorbing states at $\{\cdot, 0, \ldots, 0, \cdot\}$ (those states corresponding to there being no infected individuals remaining). Here we have used $\left(s, i_{1}, \ldots, i_{k}, r\right)$ to represent a possible realisation of $\mathbf{Y}_{t}$. For our $\mathrm{SI}(\mathrm{k}) \mathrm{R}$ epidemic with $\operatorname{Gamma}(k, \beta)$ infectious period, there are only two parameters, $\beta$ and $\gamma$, which are the disease transmission and recovery rates respectively. The nonzero transition rates of the process are

$$
\begin{aligned}
q\left(\left(s, i_{1}, \ldots, i_{k}\right),\left(s-1, i_{1}+1, \ldots, i_{k}\right)\right) & =\beta s \sum_{j=1}^{k} i_{j} \\
q\left(\left(s, i_{1}, i_{2}, \ldots, i_{k}\right),\left(s, i_{1}-1, i_{2}+1, \ldots, i_{k}\right)\right) & =\gamma i_{1} \\
q\left(\left(s, \ldots, i_{l}, i_{l+1}, \ldots, i_{k}\right),\left(s, \ldots, i_{l}-1, i_{l+1}+1, \ldots, i_{k}\right)\right) & =\gamma i_{l} \\
q\left(\left(s, \ldots, i_{k}\right),\left(s, \ldots, i_{k}-1\right)\right) & =\gamma i_{k} .
\end{aligned}
$$

\section{Diffusion Approximations}

We make use of Gaussian diffusion approximations of density dependent epidemic models, which allow us to approximate the likelihood when the number of organisms in our experiments $N$ is relatively large. The theory is presented for multidimensional processes, but we will see that there is considerably simplification in the one-dimensional case. The approximation is based on the work of Kurtz (1970, 1971), McNeil and Schach (1973) and 
Barbour $(1972,1974)$. A $d$-dimensional Markov process $\mathbf{Y}_{t}^{(N)}$ is density dependent, with index parameter $N$, if the transition rates can be written as

$$
q(\boldsymbol{y}, \boldsymbol{y}+\boldsymbol{l} ; t)=N f\left(\frac{\boldsymbol{y}}{N}, \boldsymbol{l} ; t\right) \quad\left(\boldsymbol{y}, \boldsymbol{l} \in \mathbb{Z}^{d}, \boldsymbol{l} \neq 0, t \geq 0\right)
$$

So, they depend on the state $\boldsymbol{y}$ of the process only through the population density $\boldsymbol{y} / N$, but may depend on $t$ (this is the setup of Barbour (1972)). Typically, in an experimental epidemic, the index parameter is simply the population ceiling.

In the time-inhomogoeneous case, where the transition rates do not depend on $t$, Kurtz (1970) proved a functional law of large numbers for density dependent Markov processes, and this was extended to the present timeinhomogoeneous case by Barbour (1974): as $N \rightarrow \infty$, the density process $\mathbf{X}_{t}^{(N)}:=\mathbf{Y}_{t}^{(N)} / N$ converges to a unique deterministic trajectory $\boldsymbol{x}(t)$, with $\boldsymbol{x}(0)=\boldsymbol{x}_{0}$ and satisfying the system of $d$ first-order ODEs $\partial \boldsymbol{x}(t) / \partial t=$ $F(\boldsymbol{x}(t) ; t)$, where $F(\boldsymbol{x} ; t)=\sum_{\boldsymbol{l}} \boldsymbol{l} f(\boldsymbol{x}, \boldsymbol{l} ; t)(\boldsymbol{x} \in E)$. Convergence is subject to certain technical conditions, which are satisfied for many biological models of interest, including all of the epidemic models considered here. See Appendix A for a detailed explanation.

Additionally, Kurtz (1971) and Barbour (1974) showed that the fluctuations of the density process about the deterministic trajectory, when appropriately scaled, converge over finite time intervals to a limiting Gaussian diffusion $\mathbf{Z}_{t}$ (see also Pollett $(1990,2001)$ ). Let $\mathbf{Z}_{t}^{(N)}=\sqrt{N}\left(\mathbf{X}_{t}^{(N)}-\boldsymbol{x}(t)\right)$ be the scaled density process. Then, provided $\lim _{N \rightarrow \infty} \sqrt{N}\left(\mathbf{X}_{0}^{(N)}-\boldsymbol{x}_{0}\right)=\boldsymbol{z}$ (exists), $\mathbf{Z}_{t}^{(N)}$ converges weakly in the space of all sample paths on finite time intervals to a Gaussian diffusion $\mathbf{Z}_{t}$ with initial value $\boldsymbol{z}$. There are simple 
expressions for the expectation and cross-covariance of $\mathbf{Z}_{t}: \mathbb{E}\left(\mathbf{Z}_{t}\right)=\mathbf{M}_{t} \boldsymbol{z}$ and

$$
\operatorname{Cov}\left(\mathbf{Z}_{t}, \mathbf{Z}_{t+s}\right)=\mathbf{M}_{t} \int_{0}^{t} \mathbf{M}_{u}^{-1} \mathbf{G}(\boldsymbol{x}(u) ; u)\left(\mathbf{M}_{u}^{-1}\right)^{\top} d u \mathbf{M}_{t+s}^{\top}
$$

where $\mathbf{M}_{t}=\exp \left(\int_{0}^{t} \mathbf{H}(\boldsymbol{x}(u) ; u) d u\right)(\exp (\cdot)$ denotes the matrix exponential), $\mathbf{H}(\boldsymbol{x} ; t)$ is the Jacobian matrix of $F(\boldsymbol{x} ; t)$, with entries $h_{i j}=\partial F_{j}(\boldsymbol{x} ; t) / \partial x_{k}$, and $\mathbf{G}(\boldsymbol{x} ; t)$ is the matrix with entries $g_{i j}(\boldsymbol{x} ; t)=\sum_{\boldsymbol{l}} l_{i} l_{j} f(\boldsymbol{x}, \boldsymbol{l} ; t)$. When the initial condition is fixed as part of the design for an experimental epidemic we will have $\boldsymbol{z}=\mathbf{0}$.

For experimental epidemics these results are very useful for approximating the likelihood of a series of observations. Suppose we make $n$ observations of the density process $\mathbf{X}_{t}^{(N)}$, denoting the random variables $\mathbf{X}^{\text {obs }}=$ $\left(\mathbf{X}_{1}^{\text {obs }}, \ldots, \mathbf{X}_{n}^{\text {obs }}\right)$ and the corresponding observations $\boldsymbol{x}^{\text {obs }}=\left(\boldsymbol{x}_{1}^{\text {obs }}, \ldots, \boldsymbol{x}_{n}^{\text {obs }}\right)$, where $\boldsymbol{x}_{i}^{\text {obs }}=\left(x_{1, t_{i}}^{\text {obs }}, \ldots, x_{d, t_{i}}^{\text {obs }}\right)$ so that $\boldsymbol{x}^{\text {obs }}$ has length $n d$. Furthermore, let $\boldsymbol{x}=\left(\boldsymbol{x}_{1}, \ldots, \boldsymbol{x}_{n}\right)$, where $\boldsymbol{x}_{i}=\left(x_{1}\left(t_{i}\right), \ldots, x_{d}\left(t_{i}\right)\right)$ is the vector value of the limiting deterministic trajectory at $t_{i}$. The pdf of $\mathbf{X}^{\text {obs }}$ can be approximated using a multivariate normal distribution: $\mathbf{X}^{\text {obs }} \sim \operatorname{MVN}\left(\boldsymbol{x}, \frac{1}{N} \boldsymbol{\Sigma}\right)$. Since $\mathbf{Y}_{t}^{(N)}=N \mathbf{X}_{t}^{(N)}$, we can approximate the likelihood for a series of observations $\mathbf{y}$ from the epidemic using the approximation $\mathbf{Y} \sim \operatorname{MVN}(N \boldsymbol{x}, N \boldsymbol{\Sigma})$. Here $\boldsymbol{\Sigma}$ is the cross-covariance matrix having block structure

$$
\boldsymbol{\Sigma}=\left(\begin{array}{cccc}
\boldsymbol{\Sigma}_{1,1} & \boldsymbol{\Sigma}_{1,2} & \ldots & \boldsymbol{\Sigma}_{1, n} \\
\boldsymbol{\Sigma}_{2,1} & \boldsymbol{\Sigma}_{2,2} & \ldots & \boldsymbol{\Sigma}_{2, n} \\
\vdots & \vdots & \ddots & \vdots \\
\boldsymbol{\Sigma}_{n, 1} & \boldsymbol{\Sigma}_{n, 2} & \ldots & \boldsymbol{\Sigma}_{n, n}
\end{array}\right)
$$

where $\boldsymbol{\Sigma}_{i, j}:=\operatorname{Cov}\left(\mathbf{Z}_{t_{i}}, \mathbf{Z}_{t_{j}}\right)$ is the $d \times d$ cross-covariance matrix calculated according to $(3)$. 
In the case of the (one-dimensional) time-homogeneous $(\lambda=0)$ SIS epidemic,

$$
x(t)=\frac{x_{0} x_{e q}}{x_{0}+\left(x_{e q}-x_{0}\right) e^{-\alpha t}},
$$

where $x_{e q}=1-\rho$, is the deterministic trajectory tracked by $X_{t}^{(N)}$ (the proportion of infected individuals). Variances and covariances are calculated as:

$$
\Sigma_{i, j}= \begin{cases}V\left(t_{i}\right) & (i=j) \\ V\left(t_{i}\right)\left(\frac{x_{0}+\left(x_{e q}-x_{0}\right) e^{-\alpha t_{j}}}{x_{0}+\left(x_{e q}-x_{0}\right) e^{-\alpha t_{i}}}\right)^{2} e^{-\alpha\left(t_{j}-t_{i}\right)} & (i<j)\end{cases}
$$

where

$$
\begin{gathered}
V(t)=\quad x_{0} x_{e q}\left[( 1 + \rho ) \left(x_{0}^{3}\left(e^{2 \alpha t}-1\right)+2\left(x_{e q}-x_{0}\right)^{3}\left(1-e^{-\alpha t}\right)\right.\right. \\
\left.-6 x_{0}^{2}\left(x_{e q}-x_{0}\right)\left(1-e^{\alpha t}\right)+6 x_{0} \alpha t\left(x_{e q}-x_{0}\right)^{2}\right) \\
\left.-x_{0} x_{e q}\left(x_{0}^{2}\left(e^{2 \alpha t}-1\right)-4 x_{0}\left(x_{e q}-x_{0}\right)\left(1-e^{\alpha t}\right)+2 t \alpha\left(x_{e q}-x_{0}\right)^{2}\right)\right] \\
\times\left[2(1-\rho) e^{2 \alpha t}\left(x_{0}+\left(x_{e q}-x_{0}\right) e^{-\alpha t}\right)^{4}\right]^{-1} .
\end{gathered}
$$

It is not always possible to obtain explicit analytical expressions for the entries of $\boldsymbol{x}$ and $\boldsymbol{\Sigma}$ as we have done above. This is true for the SI(k)R and many other multi-dimensional epidemic models, where numerical solution of the system of first order ODEs is required to obtain the limiting deterministic trajectory. In the case of the time-inhomogeneous SI and SIS epidemics defined by (1) with $\lambda>0$, simple analytical expressions are available for the deterministic trajectory, but the expression for the covariances is far to long to be written out here. In any case, the cross-covariance of observation vectors can be obtained easily by numerically integrating (3). Whilst numerical solution of these equations is obviously not as computationally efficient as evaluating explicit analytical expressions, the methodology presented here 
can be used to find optimal experimental designs in a matter of minutes using a package such as Matlab.

For the poSI $(\mathrm{k}) \mathrm{R}$ epidemic, our inability to observe anything other than $I_{\text {Total }}$ at each observation time requires us to make some modifications to $\boldsymbol{\Sigma}$ and $\boldsymbol{x}$. We first compute $\boldsymbol{\Sigma}$ and $\boldsymbol{x}$ as if all dimensions of the process were being observed at each observation. It is then necessary to delete all of the entries from $\boldsymbol{\Sigma}$ and $\boldsymbol{x}$ that correspond to the unobserved dimensions of the process. Finally, we sum the $k$ elements of $\boldsymbol{x}$ that correspond to the deterministic trajectories of infectious classes and also over the $k^{2}$ elements of $\Sigma$ that correspond to the variances/covariances between infectious classes. This reduces $\boldsymbol{\Sigma}$ to the $n \times n$ covariance matrix of the random variables $I_{\text {Total }, t_{1}}, \ldots, I_{\text {Total }, t_{n}}$. Similarly, $\boldsymbol{x}$ is reduced to the vector of length $n$, whose entries are the population densities of infected individuals at times $t_{1}, \ldots, t_{n}$ from the deterministic trajectory.

A computationally efficient method for calculating the likelihood of the observed data from the quantities $\boldsymbol{x}$ and $\boldsymbol{\Sigma}$ is outlined in Appendix B of Ross et al. (2009). The results are useful since the Markov property allows the multivariate normal likelihood to be written as a product of $n d$-dimensional Gaussian probability density functions. These density functions are approximations of transition density functions, conditional on previous states. The method is particularly useful for computing the approximate likelihood when the number of observations $n$ and/or the dimension of the epidemic model $d$ is large. It also facilitates efficient computation of Fisher information matrices, since these can be written as the sum of $n$ independent matrices, with each component corresponding to one of the $d$-dimensional transition den- 
sities. For relatively small values of $n d$, it is much simpler to compute the log-likelihood of the observations $\boldsymbol{y}$ as

$$
\begin{aligned}
\ell(\boldsymbol{y} ; \boldsymbol{\theta})=- & \frac{n}{2} \log (2 \pi)-\frac{1}{2} \log (|N \boldsymbol{\Sigma}(\boldsymbol{\theta})|) \\
& -\frac{1}{2 N}(\boldsymbol{y}-N \boldsymbol{x}(\boldsymbol{\theta})) \boldsymbol{\Sigma}(\boldsymbol{\theta})^{-1}(\boldsymbol{y}-N \boldsymbol{x}(\boldsymbol{\theta}))^{\top}
\end{aligned}
$$

where the dependence of $\boldsymbol{x}$ and $\boldsymbol{\Sigma}$ on $\boldsymbol{\theta}$ has been made explicit. The Fisher information matrix $\mathcal{I}$ for our Gaussian process (see Patan and Bogacka (2007)) has entries

$$
\begin{aligned}
\mathcal{I}_{i, j}= & \underbrace{N\left(\frac{\partial \mathbf{x}(\boldsymbol{\theta})}{\partial \boldsymbol{\theta}_{i}}\right) \boldsymbol{\Sigma}(\boldsymbol{\theta})^{-1}\left(\frac{\partial \mathbf{x}(\boldsymbol{\theta})}{\partial \boldsymbol{\theta}_{j}}\right)^{\top}}_{\mathcal{I}_{i, j}^{(1)}} \\
& +\underbrace{\frac{1}{2} \operatorname{tr}\left(\boldsymbol{\Sigma}(\boldsymbol{\theta})^{-1}\left(\frac{\partial \boldsymbol{\Sigma}(\boldsymbol{\theta})}{\partial \boldsymbol{\theta}_{i}}\right) \boldsymbol{\Sigma}(\boldsymbol{\theta})^{-1}\left(\frac{\partial \boldsymbol{\Sigma}(\boldsymbol{\theta})}{\partial \boldsymbol{\theta}_{j}}\right)\right)}_{\mathcal{I}_{i, j}^{(2)}}
\end{aligned}
$$

which are increasingly dominated by $\mathcal{I}_{i, j}^{(1)}$ as $N \rightarrow \infty$.

\section{Optimal Design of Experimental Epidemics}

We assume that, in an experimental epidemic, the practitioner controls: (i) the times at which the epidemic is observed, (ii) the initial conditions of the epidemic (the numbers of susceptible and infected individuals at $t_{0}=$ 0), and (iii) the number of replicate epidemics. Here a replicate epidemic consists of a closed susceptible laboratory population into which infected individuals are introduced. Our designs are of the form $\left(\boldsymbol{x}_{0}, r, \boldsymbol{t}\right)$, where $\boldsymbol{x}_{0}=\left(x_{0,1}, \ldots, x_{0, d}\right) \in(0,1]^{d}\left(\sum_{i=1}^{d} x_{0, i}=1\right)$ is the initial condition, $r$ is the number of replicate epidemics and $t_{1}<\ldots,<t_{n} \leq t_{\max }\left(t_{i} \in \boldsymbol{t}\right)$ are the 
times at which the epidemic is observed. Here, $\left[0, t_{\max }\right]$ represents the available time over which the experiment is conducted. Experimental constraints might also dictate an upper limit on the number of replicate epidemics $r$ that can be run. Since the determinant of the Fisher information matrix increases monotonically with $N$ (for fixed $\boldsymbol{t}$ and $\boldsymbol{x}_{0}$ ), the total population size should be as large as experimental resources will permit. Let $N_{\max }$ denote the maximum number of organisms available for the experiment. We shall consider only those designs where we arrange the $N_{\max }$ individuals into $r$ replicate populations of equal size, each having $N_{\max } / r$. Since each of the $r$ replicate experiments is independent, the Fisher information matrix for such a design is simply $\mathcal{I}\left(\left(x_{0}, r, \boldsymbol{t}\right) ; \boldsymbol{\theta}\right)=r \mathcal{I}\left(\left(x_{0}, 1, \boldsymbol{t}\right) ; \boldsymbol{\theta}\right)$.

We aim to find $D$-optimal experimental designs so that observations of the epidemic result in the most precise estimates of the parameters. Let $g$ be an experimental design in the set of possible designs $\mathcal{G}$. Then a $D$-optimal design $g_{\text {opt }}$ satisfies

$$
g_{\text {opt }}=\underset{g \in \mathcal{G}}{\operatorname{argmax}} \operatorname{det}(\mathcal{I}(g ; \boldsymbol{\theta})) .
$$

As mentioned previously, $g_{\text {opt }}$ is locally optimal (Chernoff, 1953), since $\mathcal{I}$ is a nonlinear function of $\boldsymbol{\theta}$. Design construction therefore relies either on some prior information about the parameters (Pronzato and Walter, 1985, 1988) or an educated guess at their values. The robustness of designs with respect to the initial guess is considered towards the end of the paper. Typically, a $D$-optimal design $g_{\text {opt }}$ is compared to another design $g$ using $D$-efficiency (see Atkinson et al. (2007)):

$$
D_{e}=\left(\frac{\operatorname{det}(\mathcal{I}(g, \boldsymbol{\theta}))}{\operatorname{det}\left(\mathcal{I}\left(g_{\text {opt }}, \boldsymbol{\theta}\right)\right)}\right)^{\frac{1}{p}}
$$


where $p$ is the dimension of $\boldsymbol{\theta}$.

In some situations, we may only be interested in estimating a subset of the parameters $\boldsymbol{\theta}_{s} \subset \boldsymbol{\theta}$ of a model, with the other parameters considered nuisance parameters. Such designs are referred to as $D_{s}$-optimal designs and are essentially the same as the $D$-optimal design, but where $\mathcal{I}$ is restricted to terms involving only partial derivatives with respect to the elements of $\boldsymbol{\theta}_{s}$. We use $D_{s}$-optimal designs (Atkinson et al., 2007) to estimate $(\beta, \mu)$ for the SIS epidemic defined in (1) where it is believed that the transmission rates are time-homogeneous $(\lambda=0)$.

We use a stochastic optimization method known as the Cross-Entropy (CE) method (Rubinstein and Kroese, 2004) for obtaining optimal designs. We generate random designs of the form $\left(\mathbf{X} ; T_{1}, \ldots, T_{n}\right)$ with $0 \leq T_{1}<$ $T_{2}<\cdots<T_{n} \leq t_{\max }$ and initial condition $\mathbf{X} \in(0,1]^{d}$, where $\sum_{i=1}^{d} X_{i}=$ 1. Each observation time $T_{i}(i=1, \ldots, n)$ and initial condition $X_{i}(i=$ $1, \ldots, d-1)$, is drawn from a beta probability density function with its own set of parameters. At each iteration, we select schedules satisfying $\operatorname{det}(\mathcal{I}) \geq$ $\gamma$ (where $\gamma$ is chosen as, say, the 90th percentile of the objective function evaluations $\left.\operatorname{det}(\mathcal{I})_{1}, \ldots, \operatorname{det}(\mathcal{I})_{m}\right)$. The selected schedules are used to update the parameters for each of the beta densities and the process is repeated until convergence is achieved. A detailed description of the algorithm is provided in Appendix B.

For the SIS and SI epidemics, the partial derivatives needed for computing $\mathcal{I}$ can be obtained analytically, but the expressions are long, so for brevity we assume that these terms are calculated numerically where required. For the poSI $(\mathrm{k}) \mathrm{R}$ epidemic, the partial derivatives must be calculated numerically. 


\section{Design Performance}

We begin by comparing the performance of three designs for each of the time-inhomogeneous SI, time-homogeneous SIS and poSI $(k) R$ epidemics via a Monte Carlo study. Tables 1 and 2 detail the parameters and designs for each of these epidemic models. Figure 1 depicts the $D$-optimal designs (initial conditions and observation times) for these three models, assuming $r=1$. The three designs considered were: (i) the $D$-optimal design, (ii) the $D$ optimal, equidistant design (where the times between successive observations are constrained to be equal), and (iii) a simple equidistant design, where the the initial number of infected individuals was set to 1 , the observation times were equally spaced and the last observation made at $t_{\max }$. For all three models we chose $t_{\max }=10$.

Table 2 summarizes our findings from 10,000 simulations of each model with each design. It is clear that the $D$-optimal design can offer a significant improvement in the accuracy and precision of the maximum likelihood estimator compared to the other two designs for the three models considered. For all three models, the $D$-optimal equidistant design performs almost as well as the $D$-optimal design and may be simpler to implement in practice. Simple equidistant designs typically perform relatively poorly and, in the case of the SIS epidemic, performance is abysmal, with extreme bias and imprecision in the maximum likelihood estimator. Such results arise when observations miss the initial drift phase of the process of this model. In general, coverage probabilities for the simple equidistant designs demonstrate that the confidence regions computed under these designs are often unreliable.

Our optimal designs detailed in Table 2 (including optimal initial condi- 
Table 1: Parameter sets used for main SI, SIS and poSI(k)R epidemic examples.

\begin{tabular}{|lcc|}
\hline Model & $\boldsymbol{\theta}$ & Composition of $\boldsymbol{x}_{0}$ \\
\hline $\mathrm{SI}$ & $(\beta, \lambda)=(1.0,0.5)$ & $x_{0}=i_{0}$ \\
\hline $\operatorname{SIS}(\lambda=0)$ & $(\beta, \mu)=(4.0,1.0)$ & $x_{0}=i_{0}$ \\
\hline $\operatorname{poSI}(4) \mathrm{R}$ & $(\beta, \gamma)=(4.0,4.0)$ & $\boldsymbol{x}_{0}=\left(s_{0}, i_{0}\right)$ \\
\hline
\end{tabular}

Table 2: $D$-optimal $/ D_{s}$-optimal and alternative designs $(n=3, N=1000)$ for the SI, SIS and $\operatorname{poSI}(\mathrm{k}) \mathrm{R}$ epidemic models listed in Table 1.

\begin{tabular}{|lccc|}
\hline Model & Design & $\boldsymbol{t}$ & $\boldsymbol{x}_{0}$ \\
\hline \multirow{3}{*}{ SI } & $D$-opt. & $(1.94,4.57,10.00)$ & 0.224 \\
& $D$-opt. eq. & $(3.33,6.67,10.00)$ & 0.209 \\
& Simp. eq. & $(3.33,6.67,10.00)$ & 0.001 \\
\hline \multirow{4}{*}{ SIS } & $D_{\text {s-opt. }}$ & $(1.45,5.99,10.00)$ & 0.042 \\
& $D_{s}$-opt. eq. & $(1.58,3.16,4.74)$ & 0.034 \\
& Simp. eq. & $(3.33,6.67,10.00)$ & 0.005 \\
\hline \multirow{3}{*}{ poSI(4)R } & $D$-opt. & $(1.11,2.01,3.50)$ & $(0.948,0.052,0,0,0)$ \\
& $D$-opt. eq. & $(1.03,2.06,3.09)$ & $(0.938,0.062,0,0,0)$ \\
& Simp. eq. & $(3.33,6.67,10.00)$ & $(0.995,0.005,0,0,0)$ \\
\hline
\end{tabular}



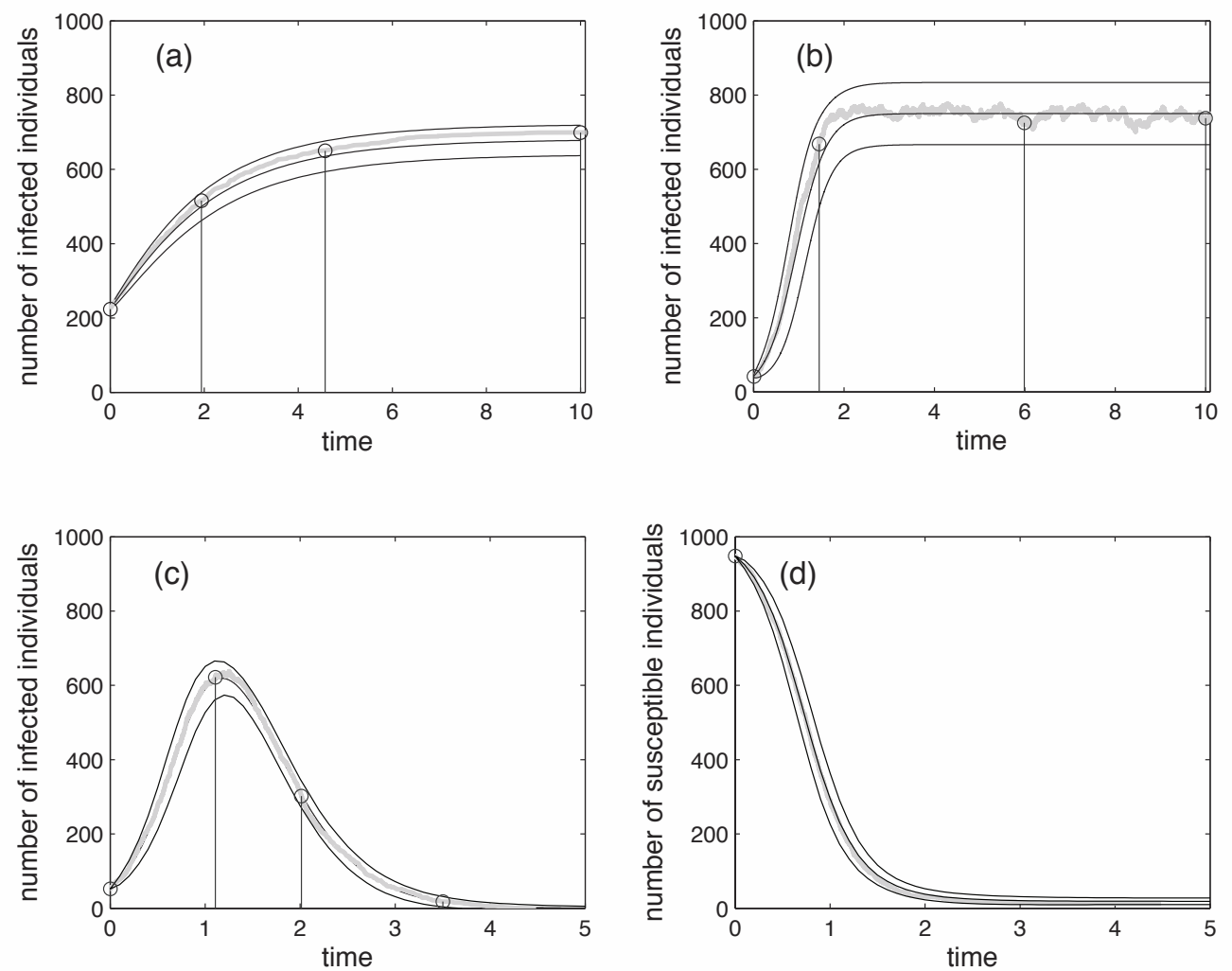

Figure 1: $D$-optimal designs $(n=3, N=1000)$ for the models listed in Table 1 and simulated (grey) trajectories (shown with limiting deterministic trajectory \pm 2 standard deviations as black lines) for: (a) the SI epidemic $(n=3)$; (b) the SIS epidemic $(n=3)$; (c and d) the poSI(4)R epidemic $(n=3)$. 
Table 3: Properties of the maximum likelihood estimator for models listed in Table 1 with corresponding designs listed in Table 2 .

\begin{tabular}{|c|c|c|c|c|}
\hline Model & Design & $\begin{array}{c}\text { 95\% Conf.Reg. } \\
\text { Cover. Prob. }\end{array}$ & $\operatorname{Bias}(\hat{\boldsymbol{\theta}})$ & Std. Dev. $(\hat{\boldsymbol{\theta}})$ \\
\hline \multirow{3}{*}{ SI } & D-opt. & 0.952 & $(1.85 \mathrm{E}-3,1.18 \mathrm{E}-3)$ & $(7.35 \mathrm{E}-2,2.94 \mathrm{E}-2)$ \\
\hline & $D$-opt. eq. & 0.952 & $(4.94$ E-3, 1.98 E-3) & $(7.66 \mathrm{E}-2,3.01 \mathrm{E}-2)$ \\
\hline & Simp. eq. & 0.624 & $(0.7198,1.71967)$ & $(1.83,2.72)$ \\
\hline \multirow{3}{*}{ SIS } & $D_{s}$-opt. & 0.934 & $(1.00 \mathrm{E}-2,4.16 \mathrm{E}-4)$ & $(0.23,0.0114)$ \\
\hline & $D_{s}$-opt. eq. & 0.949 & $(2.36 \mathrm{E}-2,-2.07 \mathrm{E}-5)$ & $(0.232,1.15 \mathrm{E}-2)$ \\
\hline & Simp. eq. & 0.953 & $(3.72,-6.34 \mathrm{E}-4)$ & $(4.86,1.01 \mathrm{E}-2)$ \\
\hline \multirow{3}{*}{$\operatorname{poSI}(4) \mathrm{R}$} & $D$-opt. & 0.93 & $(1.85 \mathrm{E}-2,1.50 \mathrm{E}-3)$ & $(0.191,8.67 \mathrm{E}-2)$ \\
\hline & $D$-opt. eq. & 0.921 & $(5.05 \mathrm{E}-4,2.01 \mathrm{E}-3)$ & $(0.188,8.43 \mathrm{E}-2)$ \\
\hline & Simp. eq. & 0.003 & $(5.60,-1.54)$ & $(1.35,0.356)$ \\
\hline
\end{tabular}


tions) were identified using our CE method (detailed in Appendix B), however, we also conducted exploratory analyses to identify how important the initial conditions were to the optimality of a design. To investigate this, the precision of the maximum likelihood estimator was studied by looking at $D$-optimal designs $(n=3, r=1)$ for the SIS epidemic. Figure 2 shows the dramatic loss of precision that can occur when $x_{0}$ is varied over a number of values between 0.02 and 0.95 and compares the distributions of the maximum likelihood estimators. For each of the initial conditions, 1000 SIS epidemics were simulated and maximum likelihood estimates obtained under the corresponding $D$-optimal design. A two-dimensional kernel density estimation routine for Matlab (Botev et al., 2010) was then used to compare the maximum likelihood estimators under these designs. The parameter values chosen were $x_{e q}=0.75, \alpha=3.0$ and $\rho=0.25$. We observe that the estimator behaves particularly poorly when $x_{0}=x_{e q}$. This is perhaps not surprising, since no drift towards equilibrium is observed, providing little information about $\alpha$. Figure 3 depicts the change in the $D$-efficiency as a function of $x_{0}$ for $D$-optimal designs under a number of different parameters. Typically, the worst designs are those where $x_{0}=x_{e q}$, however, we also observe a loss of precision when $x_{0}$ is allowed to stray close to 0 . The observed results are a consequence of very small values of $x_{0}$ generally resulting in larger variances for $\Sigma$, rather than as a result of there being a greater probability of the process being absorbed, although the latter point highlights an additional reason to avoid such designs.

Figure 4 shows the change in $D$-efficiency as $s_{0}$ (the initial proportion susceptible) is varied for the poSI(4)R epidemic. To demonstrate the accu- 

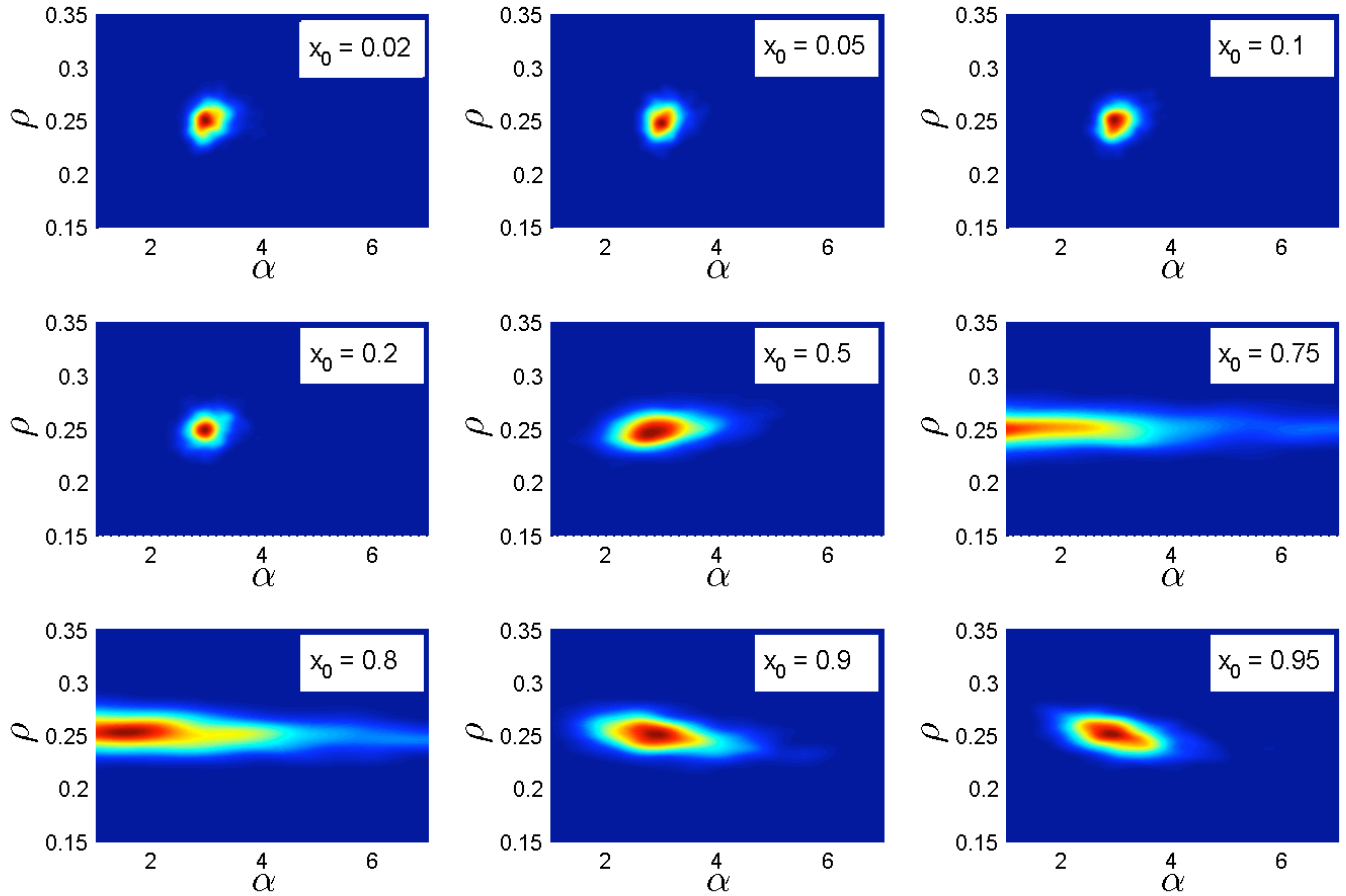

Figure 2: Density estimates for the maximum likelihood estimator using 1000 simulated trajectories of the SIS epidemic (as per Table 1) and point estimates derived using $D$ optimal designs $(n=3)$ for various values of $x_{0}$. 

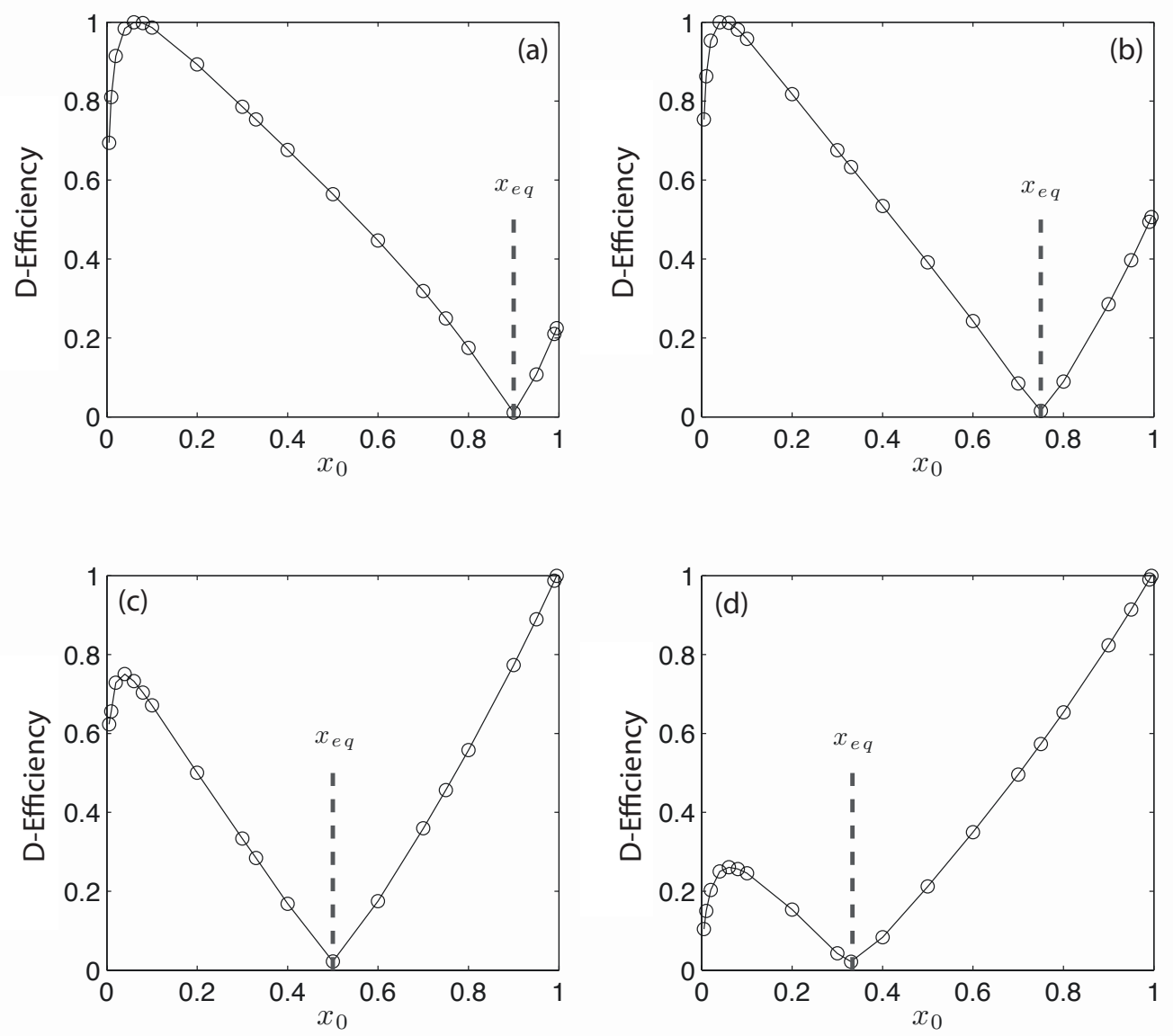

Figure 3: $D$-optimal designs $(n=3)$ obtained for various values of $x_{0}$ with $N=1000$ and different values of $\beta$ and $\mu(\lambda=0)$. D-efficiency is measured relative to the best of the optimal designs for each parameter set. 
racy of our methods in identifying optimal designs, we also include estimates of the $D$-efficiency of the design based on Monte Carlo simulations. For each of the points plotted in Figure 4, we simulated 100 trajectories of the epidemic, obtained maximum likelihood estimates under the corresponding $D$-optimal designs ( $s_{0}$ fixed) and calculated the observed Fisher information matrix $\mathcal{I}^{\text {obs }}$, having entries $\mathcal{I}_{i, j}^{\text {obs }}=-\partial^{2} \ell(\boldsymbol{y} ; \boldsymbol{\theta}) / \partial \theta_{i} \partial \theta_{j}$ evaluated at $\boldsymbol{\theta}=\hat{\boldsymbol{\theta}}(\hat{\boldsymbol{\theta}}$ being the maximum likelihood estimate). The average value of the 100 observed information matrices was then computed, denoted as $\overline{\mathcal{I}}^{\text {obs }}$ and plugged into (10) in place of $\mathcal{I}$. There is clearly very good agreement between the theoretical $D$-efficiency obtained under the diffusion approximation and the $D$-efficiency obtained through simulation, and it appears that our method is highly effective at identifying the optimal initial conditions.

We now turn attention to the issue of whether to run one large or several smaller experimental epidemics. Notice that the elements of $\mathcal{I}$ have two additive components, $\mathcal{I}_{i, j}^{(1)}$ being $O(N)$, and $\mathcal{I}_{i, j}^{(2)}$ being $O(1)$. Suppose there was a pool of 1000 individuals available for an experimental SI epidemic and 2 possible designs were under consideration: (i) a design that uses one large epidemic having $N=1000$, and (ii) a design that uses 2 replicate epidemics, each having $N=500$. Suppose also that $x_{0}$ and $\boldsymbol{t}$ are chosen so as to be optimal for the first experiment and we use the same design for both experiments. In the case of the SI epidemic, $\mathcal{I}$ is a scalar, so that for the first experiment we have $\mathcal{I}_{a}=1000 \mathcal{I}^{(1)}+\mathcal{I}^{(2)}$ and, since the Fisher information is additive for independent experiments, for the second experiment we have $\mathcal{I}_{b}=1000 \mathcal{I}^{(1)}+2 \mathcal{I}^{(2)}$. This indicates that $\mathcal{I}_{b}>\mathcal{I}_{a}$ under a design which was not even chosen to be optimal for the second experiment (so, in theory, it can 


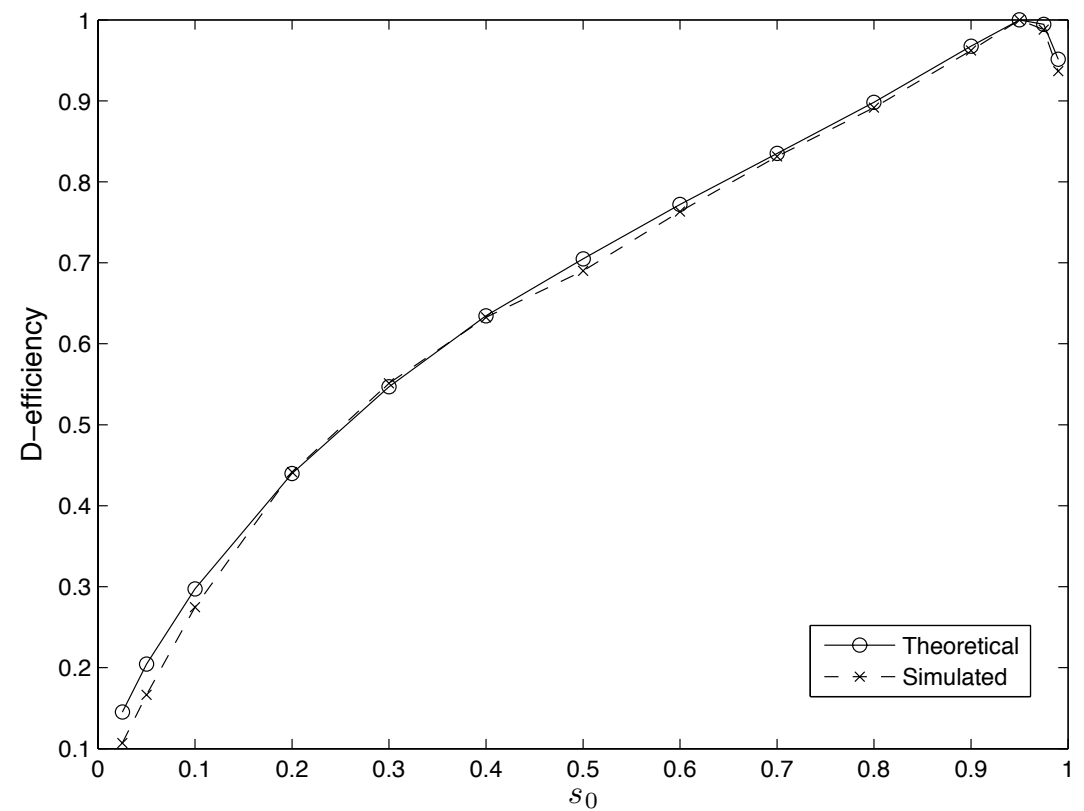

Figure 4: $D$-efficiency of $D$-optimal designs $(n=3)$ with fixed initial conditions for the poSI(4)R epidemic (as per Table 1). 
potentially do better yet). However, the argument outlined above overlooks the very important point that calculation of $\mathcal{I}$ is based on an approximation, which improves as $N \rightarrow \infty$. Thus, comparing $\mathcal{I}$ for two experiments with different values of $N$ is likely to result in errors. Figure 5 shows $D$-efficiencies of $D$-optimal designs using 1, 2, 4, 5 and 10 possible replicate epidemics from a pool of 5000 individuals for the SI, SIS and poSI(k)R epidemics. Each plot overlays the $D$-efficiency computed under the diffusion approximation with the $D$-efficiency computed by simulating 10,000 data sets for each replicate experiment for which $D$-efficiency was computed using $\overline{\mathcal{I}}_{\text {obs }}$ as before. The results highlight that the diffusion approximation should not be used for comparing the efficiency of experiments having different values of $N$. In fact, for the models considered here, the diffusion approximation gives precisely the wrong answer, since simulation suggests a small number of replicates is most efficient for all three models. In general, it appears that best practice is to use a very small number of replicate experiments, with the loss in efficiency associated with additional replicates being relatively minor.

\section{Robustness}

Finally, we consider the robustness of $D$-optimal designs to misspecification of the parameters for our three models. As mentioned previously, our $D$-optimal designs require us to have knowledge of the model parameters. Suppose that we guess the parameter $\theta$ to be $\theta_{\text {guess }}$, so the percentage error in our guess equals $100 \times \frac{\theta_{\text {guess }}-\theta}{\theta}$. Figure 6 (a) shows the relative efficiency of $D$-optimal designs created with varying amounts of error in the design parameters. For the SI epidemic, relative efficiency declines steeply if $\beta_{\text {guess }}-\beta$ 

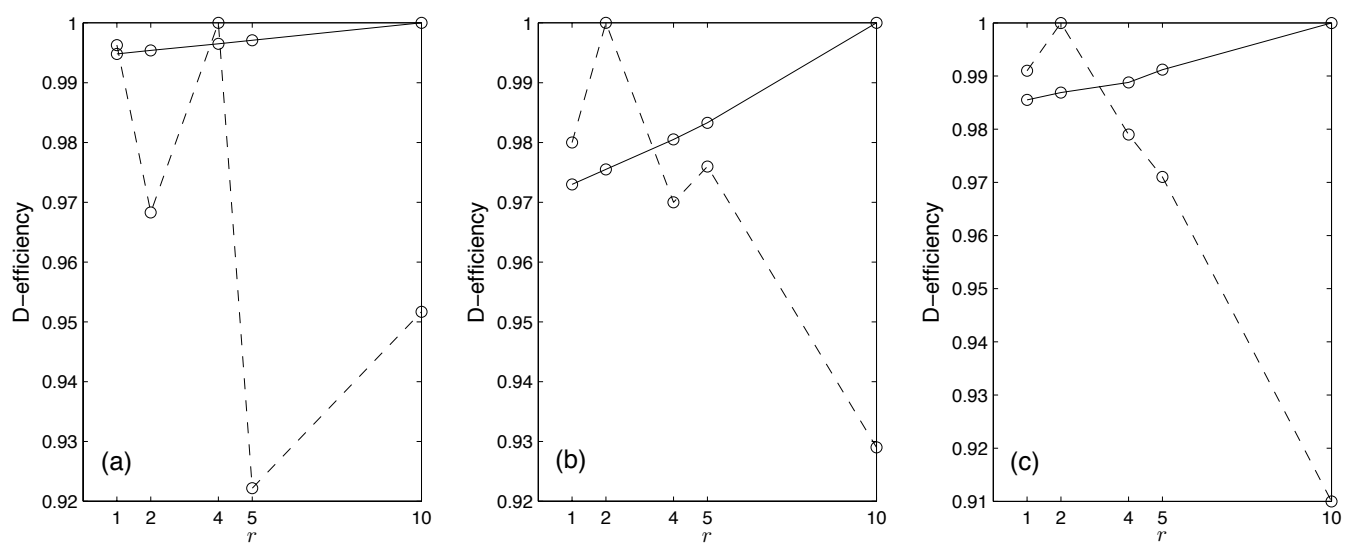

Figure 5: $D$-efficiencies of experimental epidemics $(n=2)$, with 5000 individuals divided equally between $r$ replicate experiments for: (a) the SI epidemic $(\beta=1.0, \lambda=0.5)$; (b) the SIS epidemic $(\beta=4.0, \mu=1.0, \lambda=0)$; and (c) the $\operatorname{poSI}(1) \mathrm{R}$ epidemic $(\beta=4.0, \gamma=1.0)$. Solid lines connect the values calculated under the Gaussian diffusion approximation, whilst dashed lines connect the values calculated via simulation.

is negative compared to when it is positive. This occurs because trajectories are more likely to reach the absorbing state before all observations have been taken when $\beta_{\text {guess }}$ is too small. Relative efficiency for the SIS epidemic (Figure 6 (b)) shows a similar response for the drift parameter $\alpha$, because when $\alpha_{\text {guess }}$ is too small, observations are unlikely to be scheduled during the drift phase, missing vital information about $\alpha$. Interestingly, designs for the SIS epidemic appear to be relatively insensitive to errors in $\rho$ for the values considered here. For the poSI(1)R epidemic (Figure 6 (c)), we again observe some similar behaviour to the contours of the SIS epidemic, with relative efficiency declining steeply when $\beta_{\text {guess }}-\beta$ is negative and insensitivity to error in $\gamma$. One interesting feature of Figure 6 (c) is that the relative efficiency increases in the bottom right corner, as the error in $\gamma$ becomes large and 

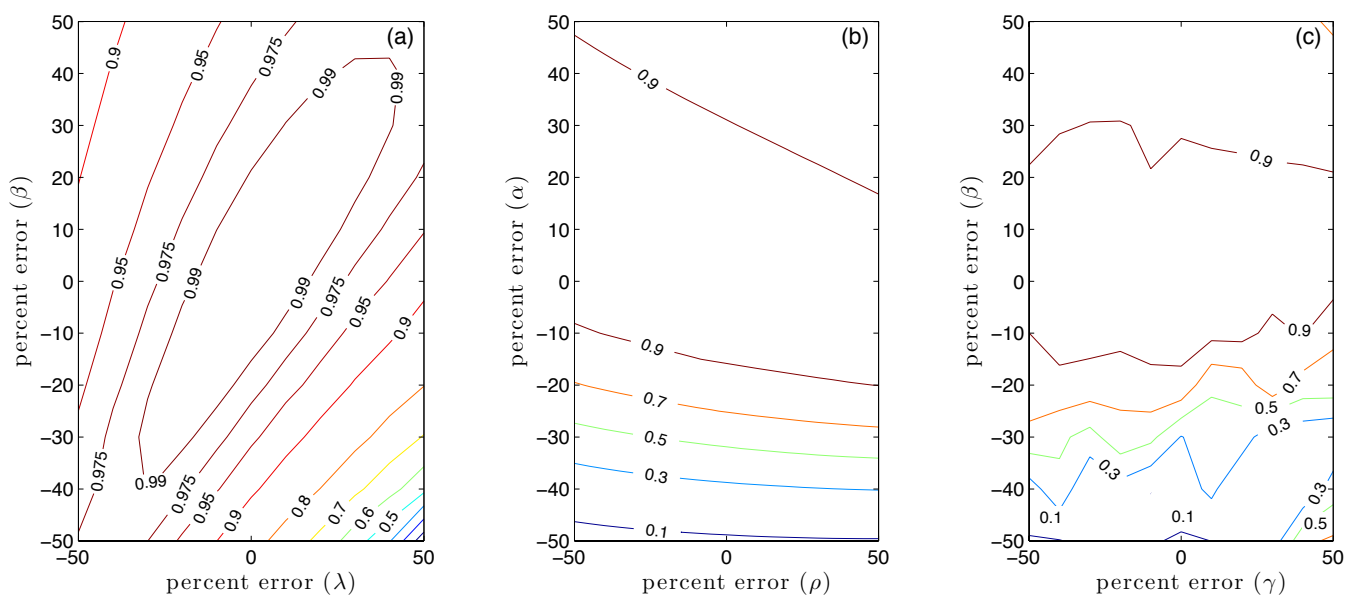

Figure 6: Relative efficiencies of optimal designs for: (a) the SI epidemic $(N=1000$, any $\beta)$ with error in $\beta_{\text {guess }}$; (b) the SIS epidemic $\left(N=1000, \alpha=3.0, \rho=\frac{1}{4}\right)$ with error in $\alpha_{\text {guess }}$ and $\rho_{\text {guess }}$ (contour plot); and (c) the $\operatorname{poSI}(1) \mathrm{R}$ epidemic $(N=1000, \beta=4.0, \gamma=1.0)$ with error in $\beta_{\text {guess }}$ and $\gamma_{\text {guess }}$ (contour plot).

positive and the error in $\beta$ becomes large and negative. This occurs because when $\beta_{\text {guess }}-\beta$ is negative, there is a tendency for observations to occur later, but when $\gamma_{\text {guess }}-\gamma$ is positive, observations tend to be taken earlier, thus the effects of the combined errors on the design are reduced to some extent and we see an increase in the relative efficiency in the lower left region of the figure.

\section{Conclusion}

We have presented a general approach to optimal experimental design for epidemics and other density dependent Markov processes. Our methods rely on the use of Gaussian diffusion approximations which are valid when the system size (usually number of individuals) is relatively large (say $N>100$ ). 
The CE method outlined in Appendix B allows for efficient calculation of these designs. Whilst we have used D-optimality throughout this exposition, our methods also work very well with other optimal design criteria, such as A-optimality and E-optimality. We have demonstrated the important effects that observation times, initial conditions and replications can have on the efficiency of such experiments through three example models: the time-inhomogeneous SI, time-homogeneous SIS and poSI $(\mathrm{k}) \mathrm{R}$ epidemics. Whilst our focus has been on these examples, the class of models to which this methodology can be applied (DDMPs) is very rich indeed. These methods and our analysis will be informative to practitioners in fields such as experimental epidemiology and biological control.

\section{Appendix A. Gaussian Diffusion Approximations}

Let $\mathbf{Y}_{t}^{(N)}(t \geq 0)$ be a $d$-dimensional continuous-time Markov process with state space $\mathcal{S}^{(N)} \subseteq \mathbb{Z}^{d}$ indexed by $N$. Let $Q(t)=\left(q(\boldsymbol{m}, \boldsymbol{n} ; t), \boldsymbol{m}, \boldsymbol{n} \in \mathcal{S}^{(N)}\right)$ be a stable and conservative set of (possibly time-dependent) transition rates; $q(\boldsymbol{m}, \boldsymbol{n} ; t)$ is the transition rate from state $\boldsymbol{m}$ to $\boldsymbol{n}(\boldsymbol{n} \neq \boldsymbol{m})$ at time $t$ and $q(\boldsymbol{m}, \boldsymbol{m} ; t):=-q(\boldsymbol{m} ; t)$, where $q(\boldsymbol{m} ; t)=\sum_{\boldsymbol{n} \neq \boldsymbol{m}} q(\boldsymbol{m}, \boldsymbol{n} ; t)(<\infty)$ is the total rate out of state $\boldsymbol{m}$ at time $t$.

Definition 1. Suppose there is an open set $E \subseteq \mathbb{R}^{d}$ and a function $f$ : $E \times \mathbb{Z}^{d} \times[0, \infty) \rightarrow \mathbb{R}$ such that

$$
q(\boldsymbol{m}, \boldsymbol{m}+\boldsymbol{l} ; t)=N f\left(\frac{\boldsymbol{m}}{N}, \boldsymbol{l} ; t\right) \quad\left(\boldsymbol{m} \in \mathcal{S}^{(N)}, \boldsymbol{l} \in \mathbb{Z}^{d}, \boldsymbol{l} \neq 0, t \geq 0\right) .
$$

\footnotetext{
Then, the family $\left\{\mathbf{Y}_{t}^{(N)}\right\}$ of Markov processes is said to be density dependent.
} 
The process $\mathbf{X}_{t}^{(N)}$, defined by $\mathbf{X}_{t}^{(N)}=\mathbf{Y}_{t}^{(N)} / N(t \geq 0)$, is called the density process. In the time-homogeneous case, where $Q$ does not depend on $t$, Kurtz Kurtz (1970) proved a functional law, which, as noted by Barbour Barbour (1974), extends to the time-inhomogeneous case. This law stipulates that, for large values of $N$, the density process 'tracks' a deterministic trajectory $\boldsymbol{x}(t)$ satisfying $d \boldsymbol{x} / d t=F(\boldsymbol{x} ; t)$, where $F: E \times[0, \infty) \rightarrow \mathbb{R}$ is given by

$$
F(\boldsymbol{x} ; t)=\sum_{\boldsymbol{l}} \boldsymbol{l} f(\boldsymbol{x}, \boldsymbol{l} ; t) \quad(\boldsymbol{x} \in E) .
$$

More precisely, we have the following result (whilst more general statements are available, the conditions given here are easy to check, and are satisfied by many biological models of interest, including all of the epidemic models considered in our paper).

Theorem 1. Suppose that, for each $\boldsymbol{l} \neq 0, f(\boldsymbol{x}, \boldsymbol{l} ; t)$ is continuous in $t$, uniformly in $\boldsymbol{x} \in E$, and that $F$ satisfies the Lipschitz condition $\mid F(\boldsymbol{x} ; t)-$ $F(\boldsymbol{y} ; t)\left|<M_{E}\right| \boldsymbol{x}-\boldsymbol{y} \mid(\boldsymbol{x}, \boldsymbol{y} \in E)$, for some constant $M_{E}$. Then, as $N \rightarrow \infty$, $\mathbf{X}_{t}^{(N)}$ converges uniformly in probability over finite time intervals $[0, t]$ to the unique deterministic trajectory satisfying

$$
\begin{gathered}
\boldsymbol{x}(0)=\boldsymbol{x}_{0}, \\
\boldsymbol{x}(s) \in E \quad(0 \leq s \leq t) \quad \text { and } \\
\frac{d}{d s} \boldsymbol{x}(s)=F(\boldsymbol{x}(s) ; s) .
\end{gathered}
$$

That is to say, for fixed $t>0$ and for all $\epsilon>0$,

$$
\lim _{N \rightarrow \infty} \mathbb{P}\left(\sup _{s \leq t}\left|\mathbf{X}_{s}^{(N)}-\boldsymbol{x}(s)\right|>\epsilon\right)=0 .
$$


Theorem 2. In addition to the conditions of Theorem 1, suppose that F has uniformly continuous first partial derivatives, and that the $d \times d$ matrix $G(\boldsymbol{x} ; t)$ with entries

$$
g_{i j}(\boldsymbol{x} ; t)=\sum_{\boldsymbol{l}} l_{i} l_{j} f(\boldsymbol{x}, \boldsymbol{l} ; t) \quad(\boldsymbol{x} \in E, t \geq 0)
$$

is bounded and uniformly continuous on $E$. If

$$
\lim _{N \rightarrow \infty} \sqrt{N}\left(\mathbf{X}_{0}^{(N)}-\boldsymbol{x}_{0}\right)=\boldsymbol{z}
$$

then $\mathbf{Z}_{s}^{(N)}(0 \leq s \leq t)$ converges weakly in $D[0, t]$ (the space of right-continuous functions with left limits on $[0, t])$ to a Gaussian diffusion $\mathbf{Z}_{s}(0 \leq s \leq t)$ with initial value $\mathbf{Z}_{0}=\boldsymbol{z}$ and characteristic function $\psi=\psi(t, \theta)$ satisfying

$$
\frac{\partial \psi}{\partial t}(t, \theta)=-\frac{1}{2} \sum_{j, k} \theta_{j} \theta_{k} g_{j k}(\boldsymbol{x}(t) ; t) \psi(t, \theta)+\sum_{j, k} \theta_{j} h_{j k}(\boldsymbol{x}(t) ; t) \frac{\partial \psi}{\partial \theta_{k}}(t, \theta),
$$

where $h_{j k}(\boldsymbol{x} ; t)=\partial F_{j}(\boldsymbol{x} ; t) / \partial x_{k}$.

Remark 2. The additional conditions of Theorem 2 will also hold if $f(\boldsymbol{x}, \boldsymbol{l} ; t)$ is multinomial. 
Appendix A of Ross et al. (2009) provides simple expressions for the expected value and covariance of $\mathbf{Z}_{t}$, which can be extended in an obvious way to the present case. Let $\mathbf{M}_{s}=\exp \left(\int_{0}^{s} \mathbf{H}(\boldsymbol{x}(u) ; u) d u\right)$, where $\mathbf{H}(\boldsymbol{x} ; t)$, the Jacobian matrix of $F(\boldsymbol{x} ; t)$, has entries $h_{j k}(\boldsymbol{x} ; t)$. Then, $\mathbb{E}\left(\mathbf{Z}_{t}\right)=\mathbf{M}_{t} \boldsymbol{z}$ and

$$
\operatorname{Cov}\left(\mathbf{Z}_{t}, \mathbf{Z}_{t+s}\right)=\mathbf{M}_{t} \int_{0}^{t} \mathbf{M}_{u}^{-1} \mathbf{G}(\boldsymbol{x}(u) ; u)\left(\mathbf{M}_{u}^{-1}\right)^{\top} d u \mathbf{M}_{t+s}^{\top} \quad(s \geq 0) .
$$

\section{Appendix B. A Cross-Entropy Method for the Optimal Design}

We employ the Cross-Entropy (CE) method of stochastic optimisation Rubinstein and Kroese (2004) for identifying optimal initial conditions and observation times. It operates in the present context by randomly generating designs of the form $\left(\mathbf{X} ; T_{1}, \ldots, T_{n}\right)$ with $0 \leq T_{1}<T_{2}<\cdots<T_{n} \leq t_{\max }$ and initial condition $\mathbf{X} \in(0,1]^{d}$, where $\sum_{i=1}^{d} X_{i}=1$, and $d$ is the dimension of the epidemic. With each step of the routine, the top performing designs are used to update the parameters of the probability distributions (from which the random designs were generated). Ideally, this updating is done using their maximum likelihood estimates, however, since there is no closed form for the parameters of the beta distribution, we approximate using the method of moments estimators. With each iteration, the means of the probability densities converge towards the values corresponding to the optimal observation times/optimal initial conditions and the variances decrease towards 0 .

Our implementation of the CE method generates observation times on the interval $\left[0, t_{\max }\right]$ and initial conditions on $(0,1]^{d}$ using beta probability distributions, each with parameter vector $\boldsymbol{\nu}_{i}=\left(\alpha_{i}, \beta_{i}\right)$. The probability distributions are initiated with parameter vectors $(1,1)$, so that the densities 
are uniform on the interval under consideration. Updating is done according to the algorithm described below, and is based upon recommendations in Rubinstein and Kroese (2004).

\section{Appendix B.1. Cross-Entropy Algorithm}

1. Define values for $t_{\max }, n, d, \delta_{\min }, y_{0}, \boldsymbol{\theta}$ and $N$.

2. Set the following parameter values controlling parameter updating: $\eta=$ $0.9, s_{1}=0.7, s_{2}=0.7$ and $s_{3}=5$. These parameters are used to ensure the optimisation routine takes a 'smooth' trajectory through the solution space and does not converge to a sub-optimal solution.

3. Set the maximum variance threshold, which is used to end the optimisation routine, to be $\epsilon=10^{-5}$.

4. Set the timestep $k=0$ and the initial parameter vectors $\boldsymbol{\theta}_{i}$ for each of $n+d-1$ beta probability density functions $f_{i}\left(x_{i} ; \boldsymbol{\nu}_{i}\right)$ for $i=1, \ldots, d-1$ and $f_{i}\left(t_{i} ; \boldsymbol{\nu}_{i}\right)$ for $i=d, \ldots, n+d-1$ as $(1,1)$.

5. Create $m$ designs $\mathbf{D}_{1, k}, \ldots, \mathbf{D}_{m, k}$, such that the $i$ th element of each design is generated from the beta probability density function $f_{i}\left(\cdot ; \alpha_{i, k}, \beta_{i, k}\right)$, where

$$
\mathbf{D}_{j, k}=\left(X_{1, j, k}, \ldots, X_{d-1, j, k} ; T_{1, j, k}, \ldots, T_{n, j, k}\right)
$$

In addition, a rejection step is introduced to retain only those designs where the observation times satisfy $0<T_{1}<\cdots<T_{n} \leq t_{\max }$ and $\sum_{i=1}^{d-1} X_{i} \leq 1$. One can also impose $T_{i}-T_{i-1} \geq \delta_{\text {min }},\left(1 \leq i \leq n ; \delta_{\text {min }} \geq\right.$ 
0 ) if desired. These observation times do not include the mandatory initial observation at $t=0$. The beta probability density functions defined on the interval $\left[0, t_{\max }\right]$ have the form:

$$
f_{i}^{(t)}\left(t_{i} ; \alpha_{i, k}^{(t)}, \beta_{i, k}^{(t)}\right)=\frac{\left(\frac{t_{i}}{t_{\max }}\right)^{\alpha_{i, k}^{(t)}-1}\left(1-\frac{t_{i}}{t_{\max }}\right)^{\beta_{i, k}^{(t)}-1}}{B\left(\alpha_{i, k}^{(t)}, \beta_{i, k}^{(t)}\right)},
$$

whilst the beta density for the the initial conditions are:

$$
f_{i}^{(x)}\left(x_{i} ; \alpha_{i, k}^{(x)}, \beta_{i, k}^{(x)}\right)=\frac{x_{i}^{\alpha_{i, k}^{(x)}-1}\left(1-x_{i}\right)^{\beta_{i, k}^{(x)}-1}}{B\left(\alpha_{i, k}^{(x)}, \beta_{i, k}^{(x)}\right)},
$$

where $B(\alpha, \beta)$ is the beta function $B(\alpha, \beta)=\Gamma(\alpha) \Gamma(\beta) / \Gamma(\alpha+\beta)$.

6. Calculate the performance (D-optimality) $p_{1, k}, \ldots, p_{m, k}$ of each of the $m$ designs by calculating the determinant of the Fisher information matrix, $\operatorname{det}\left(\mathcal{I}\left(\boldsymbol{\theta}, \mathbf{D}_{j, k}\right)\right)$.

7. Calculate the $\eta$-quantile of $p_{1, k}, \ldots, p_{m, k}$ and record this as $q_{k}$.

8. Let $D_{i, j, k}$ denote the $i$ th design point (time or initial condition) of $\mathbf{D}_{j, k}$. Calculate the mean and variance of each design point at the $k$ th time step as follows:

$$
\bar{D}_{i, k}=\left\{\begin{array}{c}
s_{1}\left(\frac{\sum_{j=0}^{m} I_{\left\{p_{j, k} \geq q_{k}\right\}} D_{i, j, k}}{\sum_{j=0}^{m} I_{\left\{p_{j, k} \geq q_{k}\right\}}}\right) \\
+\left(1-s_{1}\right) \bar{D}_{i, k-1} \quad(k \geq 1), \\
\frac{\sum_{j=0}^{m} I_{\left\{p_{j, k} \geq q_{k}\right\}} D_{i, j, k}}{\sum_{j=0}^{m} I_{\left\{p_{j, k} \geq q_{k}\right\}}} \quad(k=0),
\end{array}\right.
$$




$$
\operatorname{Var}\left(D_{i, k}\right)=\left\{\begin{array}{l}
{\left[u_{k}\left(\frac{\sum_{j=0}^{m}\left(I_{\left\{p_{j, k} \geq q_{k}\right\}} D_{i, j, k}-\operatorname{Mean}\left(D_{i, k}\right)\right)^{2}}{\sum_{j=0}^{m} I_{\left\{p_{j, k} \geq q_{k}\right\}}}\right)^{\frac{1}{2}}\right.} \\
\left.+\left(1-u_{k}\right)\left(\operatorname{Var}\left(D_{i, k-1}\right)\right)^{1 / 2}\right]^{2} \quad(k \geq 1), \\
\frac{\sum_{j=0}^{m}\left(I_{\left\{p_{j, k} \geq q_{k}\right\}} D_{i, j, k}-\operatorname{Mean}\left(D_{i, k}\right)\right)^{2}}{\sum_{j=0}^{m} I_{\left\{p_{j, k} \geq q_{k}\right\}}} \quad(k=0),
\end{array}\right.
$$

and

$$
u_{k}=s_{2}-s_{2}\left(1-\frac{1}{k}\right)^{s_{3}} .
$$

9. Update the time step: $k=k+1$.

10. Update the parameter vectors for each of the beta probability densities, in such a way that the density giving rise to the $i$ th design point has parameters

$$
\alpha_{i, k}^{(\cdot)}=\frac{\frac{r_{i, k}}{\left(1+r_{i, k}\right)^{2}}-\operatorname{Var}\left(D_{i, k}\right)}{\left(\operatorname{Var}\left(D_{i, k}\right)\left(1+r_{i, k}\right)\right)} \text { and } \quad \beta_{i, k}^{(\cdot)}=\frac{\alpha_{i, k}^{(\cdot)}-\bar{D}_{i, k} \alpha_{i, k}^{(\cdot)}}{\bar{D}_{i, k}}
$$

where $r_{i, k}=\left(1-\bar{D}_{i, k}\right) / \bar{D}_{i, k}$.

11. If $\max _{i} \operatorname{Var}\left(D_{i, k}\right) \leq \epsilon$, then return the vector $\left(\bar{D}_{1, k}, \bar{D}_{2, k}, \ldots, \bar{D}_{n+d-1, k}\right)$ or $\left(\bar{D}_{1, k}, \ldots, \bar{D}_{n, k}\right)$ and terminate the routine, otherwise return to step 5.

\section{References}

Andersson, H., Britton, T., 2000. Stochastic Epidemic Models and their Statistical Analysis. Springer-Verlag, New York.

Atkinson, A., Donev, A., Tobias, R., 2007. Optimum Experimental Designs, with SAS. Oxford University Press, Oxford. 
Bailey, D., Otten, W., Gilligan, C., 2000. Saprotrophic invasion by the soilborne fungal plant pathogen Rhizoctonia solani and percolation thresholds. New Phytologist 146, 535-544.

Bailey, N., 1950. A simple stochastic epidemic. Biometrika 37, 193-202.

Bailey, N., 1957. The Mathematical Theory of Epidemics. Griffin, London.

Barbour, A., 1972. The principle of the diffusion of arbitrary constants. Journal of Applied Probability 9, 519-541.

Barbour, A., 1974. On a functional central limit theorem for Markov population processes. Advances in Applied Probability 6, 21-39.

Bartlett, M., 1949. Some evolutionary stochastic processes. Journal of the Royal Statistical Society, Series B (Methodological) 11, 211-229.

Bartlett, M., 1956. Deterministic and stochastic models for recurrent epidemics. Proceedings of the Third Berkley Symposium on Mathematical Statistics and Probability 4, 81.

Becker, G., Kersting, G., 1983. Design problems for the pure birth process. Advances in Applied Probability 15, 255-273.

Botev, Z., Grotowski, J., Kroese, D., 2010. Kernel density estimation via diffusion. Annals of Statistics 38, 2916-2957.

Cauchemez, S., Ferguson, N., 2008. Likelihood-based estimation of continuous-time epidemic models from time-series data: application to measles transmission in London. Journal of the Royal Society Interface 5, 885-897. 
Chernoff, H., 1953. Locally optimal designs for estimating parameters. The Annals of Mathematical Statistics 24, 586-602.

Clancy, D., Mendy, S., 2011. Approximating the quasi-stationary distribution of the SIS model for endemic infection. Methodology and Computing in Applied Probability 13, 603-618.

Cook, A., Gibson, G., Gilligan, C., 2008. Optimal observation times in experimental processes. Biometrics 64, 860-868.

van der Goot, J., Koch, G., de Jong, M., van Boven, M., 2005. Quantification of the effect of vaccination on transmission of avian influenza (H7N7) in chickens. Proceedings of the National Academy of Sciences (USA) 102, $18141-18146$.

Höhle, M., Jørgensen, E., O’Neill, P., 2005. Inference in disease transmission experiments by using stochastic epidemic models. Journal of the Royal Statistical Society: Series C (Applied Statistics) 54, 349-366.

Keeling, M., 2000. Multiplicative moments and measures of persistence in ecology. Journal of Theoretical Biology 205, 269-281.

Keiding, N., 1974. Estimation in the birth process. Biometrika 61, 71-80.

Keiding, N., 1975. Maximum likelihood estimation in the birth-and-death process. The Annals of Statistics 3, 363-372.

Kendall, D., 1949. Stochastic processes and population growth. Journal of the Royal Statistical Society. Series B (Methodological) 11, 230-282. 
Kendall, D., 1956. Deterministic and stochastic epidemics in closed populations. Proceedings of the Third Berkley Symposium on Mathematical Statistics and Probability 4, 149-165.

Kermack, W., McKendrick, A., 1927. A contribution to the mathematical theory of epidemics. Proceedings of the Royal Society of London 115, $700-721$.

Kim, J., Radhakrishnan, S., 2006. Cost optimization in SIS model of worm infection. ETRI Journal 28, 692-695.

Kingman, J., 1969. Markov population processes. Journal of Applied Probability $6,1-18$.

Kleczkowski, A., Gilligan, C., Bailey, D., 1997. Scaling and spatial dynamics in plant-pathogen systems: from individuals to populations. Proceedings of the Royal Society of London, Series B 264, 979-984.

Krishnarajah, I., Cook, A., Marion, G., Gibson, G., 2005. Novel moment closure approximations in stochastic epidemics. Bulletin of Mathematical Biology 67, 855-873.

Kurtz, T., 1970. Solutions of ordinary differential equations as limits of pure jump Markov processes. Journal of Applied Probability 7, 49-58.

Kurtz, T., 1971. Limit theorems for sequences of jump Markov processes approximating ordinary differential processes. Journal of Applied Probability $8,344-356$. 
McCormack, R., Allen, L., 2007. Disease emergence in multi-host epidemic models. Mathematical Medicine and Biology 24, 17-34.

McNeil, D., Schach, S., 1973. 1973. Journal of the Royal Statistical Society, Series B (Methodological) 35, 1-23.

Nåsell, I., 2003. An extension of the moment closure method. Theoretical Population Biology 64, 233-239.

Neuts, M., 1975. Probability distributions of phase type, in: Liber Amicorum Prof. Emeritus H. Florin. University of Louvain, pp. 173-206.

O’Neill, P., Roberts, G., 1999. Bayesian inference for partially observed stochastic epidemics. Journal of the Royal Statistical Society, Series A (Statistics in Society) 162, 121-129.

Otten, W., Filipe, J., Bailey, D., Gilligan, C., 2003. Quantification and analysis of transmission rates for soilbourne epidemics. Ecology 84, 32323239 .

Pagendam, D., Pollett, P., 2009. Optimal sampling and problematic likelihood functions in a simple population model. Environmental Modelling and Assessment 14, 759-767.

Pagendam, D., Pollett, P., 2010a. Locally optimal designs for the simple death process. Journal of Statistical Planning and Inference 140, 30963105.

Pagendam, D., Pollett, P., 2010b. Robust optimal observation of a metapopulation. Ecological Modelling 221, 2515-2525. 
Patan, M., Bogacka, B., 2007. Optimum experimental designs for dynamic systems in the presence of correlated errors. Computational Statistics and Data Analysis 51, 5644-5661.

Pollett, P., 1990. On a model for interference between searching insect parasites. Journal of the Australian Mathematical Society 31, 133-150.

Pollett, P., 2001. Diffusion approximations for ecological models. In(Ed. Fred Ghassemi) Proceedings of the International Congress on Modelling and Simulation, Vol. 2, Modelling and Simulation Society of Australia and New Zealand, Canberra, Australia , 848-848.

Pronzato, I., Walter, E., 1985. Robust experimental design via stochastic approximation. Mathematical Biosciences 75, 103-120.

Pronzato, I., Walter, E., 1988. Robust experimental design via maximin optimization. Mathematical Biosciences 89, 161-176.

Ross, J., Pagendam, D., Pollett, P., 2009. On parameter estimation in population models II: multi-dimensional processes and transient dynamics. Theoretical Population Biology 75, 123-132.

Ross, J., Taimre, T., Pollett, P., 2006. On parameter estimation in population models. Theoretical Population Biology 70, 498-510.

Rubinstein, R., Kroese, D., 2004. The Cross-Entropy Method: A Unified Approach to Combinatorial Optimization, Monte-Carlo Simulation, and Machine Learning. Springer-Verlag, New York. 
Sidje, R., 1998. Expokit: A software package for computing matrix exponentials. ACM Transactions on Mathematical Software 24, 130-156.

Stone, P., Wilkinson-Herbots, H., Isham, V., 2008. A stochastic model for head lice infection. Journal of Mathematical Biology 56, 743-763.

Streftaris, G., Gibson, G., 2004. Bayesian analysis of experimental epidemics of foot-and-mouth disease. Proceedings of the Royal Society of London, Series B 271, 1111-1117.

Whittle, P., 1957. On the use of the normal approximation in the treatment of stochastic processes. Journal of the Royal Statistical Society, Series B (Methodological) 19, 268-281. 